\title{
NUCLEIC ACID (DNA) IMMUNIZATION AS A PLATFORM FOR DENGUE VACCINE DEVELOPMENT
}

Kevin R. Porter*, M. D. and Kanakatte Raviprakash, Ph. D.

Naval Medical Research Center, Infectious Diseases Directorate, Silver Spring, Maryland.

*Corresponding Author: email - kevin.r.porter3.civ@mail.mil; Phone - 301-319-7450; Fax $301-319-7460$

Running Title: Dengue DNA Vaccines

Key Words: dengue, DNA vaccines, clinical trials, non-human primates, Shuffled DNA vaccine, plasmid, Biojector

(C) 2015. This manuscript version is made available under the Elsevier user license http://www.elsevier.com/open-access/userlicense/1.0/ 


\begin{abstract}
Since the early 1990s, DNA immunization has been used as a platform for developing a tetravalent dengue vaccine in response to the high priority need for protecting military personnel deployed to dengue endemic regions of the world. Several approaches have been explored ranging from naked DNA immunization to the use of live virus vectors to deliver the targeted genes for expression. Pre-clinical animal studies were largely successful in generating anti-dengue cellular and humoral immune responses that were protective either completely or partially against challenge with live dengue virus. However, Phase 1 clinical evaluation of a prototype monovalent dengue 1 DNA vaccine expressing prM and E genes revealed anti-dengue T cell IFN $\gamma$ responses, but poor neutralizing antibody responses. These less than optimal results are thought to be due to poor uptake and expression of the DNA vaccine plasmids. Because DNA immunization as a vaccine platform has the advantages of ease of manufacture, flexible genetic manipulation and enhanced stability, efforts continue to improve the immunogenicity of these vaccines using a variety of methods.
\end{abstract}




\section{Introduction}

Dengue poses a tremendous global public health threat, occurring in approximately 100 countries [1]. Current strategies to prevent this disease include the use of mosquito repellants, vector control using insecticides, eliminating mosquito breeding sites and the use of bed nets. The effectiveness of the latter is questioned due to the day-biting nature of Aedes mosquitoes. As of the writing of this article, there are no FDA approved drugs or vaccines to treat or prevent dengue.

Given the global distribution of dengue with the circulation of multiple dengue serotypes in many different countries, the development of an effective tetravalent dengue vaccine is a top priority for U. S. military forces and public health agencies. For decades U. S. military scientists worked to develop a dengue vaccine. Investigators at the Walter Reed Army Institute of Research used traditional approaches to develop a live attenuated vaccine and currently are moving forward with a purified inactivated dengue vaccine.

Scientists at the Naval Medical Research Center (formally the Naval Medical Research Institute) pursued molecular platforms to complement the Army's dengue vaccine development programs. These efforts later were consolidated into a joint Army-Navy dengue vaccine program under the Military Infectious Diseases Research Program.

The various platforms utilized by Navy investigators as part of this joint effort primarily involved naked DNA immunization. To enhance the immunogenicity of experimental dengue DNA vaccines, several approaches were explored including DNA formulated in an 
adjuvant, virus vectored vaccines, use of immunostimulatory nucleic acid sequences and differing the routes of vaccine administration. Table 1 summarizes the different approaches. The preclinical and clinical successes and failures of each of these approaches is the subject of this review article.

\section{Naked DNA Immunization}

\section{DNA Vaccine Construct Development}

The dengue virus genome consists of three structural and seven nonstructural genes, in addition to 3' and 5' non-coding regions. The three structural genes code for capsid (C), pre-membrane (prM) and envelope (E) proteins. While immune responses are elicited primarily to the structural proteins as well as the nonstructural proteins NS1, NS3 and NS5, neutralizing antibody responses are directed primarily to epitopes on the envelope protein. Cellular immune responses are mostly generated against the nonstructural proteins. Given the epidemiological evidence in infants demonstrating that anti-dengue neutralizing antibodies appear to be sufficient to provide protection against dengue disease, early naked DNA vaccine development efforts centered on the use of the envelope gene.

The studies with dengue DNA vaccines outlined below proved the feasibility of this approach. They also informed us of the need to develop approaches and methods to improve the immunogenicity of the vaccine constructs. In the case of DNA vaccines, immunogenicity is a function of many variables such as the uptake of DNA by immunologically relevant cells, level and type of antigen expressed, and presentation of antigen to mount an immune response. 
The first series of proof-of-principle experiments focused on the development of a monovalent dengue 2 (DEN-2) DNA vaccine. The construct consisted of the prM gene and 92\% of the envelope gene of the New Guinea C DEN-2 strain, cloned into a plasmid vector provided by Vical Inc. The transmembrane portion of the E gene was omitted to promote secretion of the intracellularly expressed DEN-2 E protein. Murine studies with this vaccine demonstrated the production of anti-DEN-2 neutralizing antibodies that protected against intracerebral challenge with live DEN-2 virus [2].

Follow on work was performed to systematically determine the optimal configuration of a dengue DNA vaccine based on E protein antigen. We published murine studies showing that plasmid DNA vaccines containing dengue 1 Western Pacific 74 strain (DEN-1) prM and full length E (prM 100\%E) were optimal in producing neutralizing antibody responses [3]. In that study, plasmids containing $80 \% \mathrm{E}$, prM $92 \% \mathrm{E}$ and prM $100 \% \mathrm{E}$ resulted in the secretion of dengue E proteins into culture media when transfected into 293 cells. Plasmids expressing prM 80\%E did not adequately express DEN-1 E proteins. When the plasmids were administered to mice by intradermal (ID)/subcutaneous (SC) injection, only the $80 \% \mathrm{E}$ and prM $100 \% \mathrm{E}$ constructs generated high levels of neutralizing antibody, with the prM 100\%E plasmid producing the highest and most durable response. Analysis of the culture supernatants of transfected cells revealed that the E protein secreted into the medium of cells transfected with prM 100\%E, but not $80 \% \mathrm{E}$, was present in the form of virus-like-particles [3]. Based on these results, DNA vaccine constructs containing DEN-1 prM $100 \%$ E genes were chosen for evaluation of protective efficacy in non-human primate 
models. While there are a few other studies of dengue DNA vaccines based on E protein [4, 5] and NS-1 protein [6-8], to our knowledge none have moved beyond evaluation in animal models.

\section{Preclinical Testing for Immunogenicity and efficacy in Non-human Primates}

The DEN-1 construct was tested in both rhesus macaques and Aotus monkeys for the ability to generate a protective immune response. Protective efficacy was assessed by challenge with live DEN-1 virus and subsequent measurement of duration/magnitude of the postchallenge dengue viremia.

Mode of vaccine delivery was also assessed in these experiments. Intramuscular (IM) and subcutaneous delivery of vaccines were the mainstay because of ease of administration and the ability to deliver relatively larger volumes. However, delivery via the ID route is believed to be more effective due to the presence of antigen presenting Langerhans cells that serve as a first line of defense against infectious pathogens. The vaccine in both nonhuman studies was therefore administered both by the ID and IM routes.

In the Aotus [9] study, there was a trend toward higher antibody responses when administered intradermally, but the differences were not statistically significant [9]. Small group sizes and the presence of keratinized tissue in Aotus skin may have contributed to not fully realizing the potential of intradermal delivery. Animals immunized both ID and IM showed some level of protection against live virus challenge. 
In contrast to the results obtained using Aotus monkeys, ID immunized rhesus monkeys had poor neutralizing antibody responses [10]. The reason for the poor antibody responses in the ID immunized group was unclear, but it was thought that differences in the skin anatomy of the older rhesus macaques may have contributed. Consistent with the poor antibody response, the ID immunized animals were not protected against live virus challenge whereas the IM immunized rhesus monkeys showed partial protection.

Intradermal inoculation in humans is not favored due to limitation in the volumes that can be effectively delivered, requirement of specially trained personnel for administration and the variability associated with the procedure. In recent years, however, specialty needlefree devices have entered the market that can deliver significantly larger volumes with much ease and consistency.

Based on the results obtained with the DEN-1vaccine, similar plasmid DNA vaccine constructs for DEN-2, DEN-3 and DEN-4 were developed and contained the prM 100\% E genes of Philippine strains of DEN-2, DEN-3 and DEN-4. In vitro testing revealed that all three constructs expressed the appropriate prM and E proteins by IFA and western blot analysis, and were immunogenic in animal models including non-human primates [11](Raviprakash_unpublished). At about the same time, Putnak and colleagues of WRAIR reported immunogenicity and protective efficacy of a DEN-2 DNA vaccine delivered by gene-gun in rhesus macaques [12]. 
As indicated previously, it is necessary that a dengue vaccine provide protection against all four dengue virus serotypes. Immune responses elicited by natural infection with dengue virus are only able to protect long term against reinfection from the same serotype. Lack of long term cross protection demands that four individual vaccines, one for each serotype, be mixed to produce the final vaccine formulation. This process adds significantly to the cost and complexity of dengue vaccine development. In vaccines that are based on replicating attenuated or chimeric viruses, serotype competition or dominance can potentially affect vaccine efficacy. To address this problem and reduce dengue vaccine complexity, tetravalent dengue DNA vaccines using 'shuffled DNA' technology was explored. Using this technology, several plasmid DNA vaccines were prepared that each expressed single E proteins containing epitopes from all four dengue serotypes. A functional screen in the mouse model identified several shuffled DNA vaccine constructs that elicited neutralizing antibodies to all four serotypes in mice following four, $100 \mu \mathrm{g}$ doses given intramuscularly [13]. Three selected vaccine constructs were tested in the rhesus macaque model, where they elicited only weak to modest tetravalent antibody responses and provided incomplete protection from viremia upon virus challenge [14]. A subsequent study in which macaques were primed with a shuffled DNA vaccine and boosted using a heterologous shuffled protein antigen (in vitro expressed purified E protein containing different epitopes from all four serotypes) failed to show improved immunogenicity (Raviprakash, unpublished). These initial studies, although providing a basis for the DNA immunization approach for DEN vaccine development, highlighted the need for enhancing the immunogenicity of DNA vaccines. 


\section{Approaches to Improve DNA Vaccine Immunogenicity}

Uptake of DNA vaccines by immunologically relevant cells can be influenced by the route and method of vaccine administration, and by the inclusion of agents that have immunostimulatory effects. It is also important that the antigen expressed be folded properly and present immunological epitopes similar to those exposed in a natural dengue virus infection.

In vivo priming by DNA vaccines appears to occur predominantly by antigen transfer[15]. Although there may be some direct transfection of bone marrow derived APCs, the bulk of the immune response is due to expression of antigen in transfected non-lymphoid cells and subsequent transfer to APCs. It appears therefore, that the immune responses to a DNA vaccine can be improved either by targeting APCs for in vivo transfection, or by increasing the number of non-lymphoid cells that are transfected, or both[16].

Delivery of vaccines using needle-free jet injectors has certain advantages. In addition to removing the hazards associated with needles, this method has been shown to improve tissue distribution and enhance uptake of DNA vaccines[17]. Agents such as CpG motifcontaining immunostimulatory sequences (ISS) that improve the function of professional antigen presenting cells (APC) and granulocyte-monocyte colony stimulating factor (GMCSF) that attracts APC to the site of injection have been used as 'adjuvants' in attempts to increase the number of APCs directly transfected in vivo[18, 19]. In one study, Aotus monkeys were vaccinated with DEN-1 DNA vaccine containing ISS (pHis64 plasmid which contains 64 copies of human immunostimulatory CpG motifs) and a plasmid expressing 
Aotus specific GM-CSF. The vaccine cocktail was delivered ID either by needle and syringe or by the needle-free Biojector device. Although inclusion of ISS did not result in higher antibody responses, animals vaccinated with all three components either by needle and syringe, or Biojector did elicit higher neutralizing antibodies compared to DEN-1 DNA vaccine alone[20]. These animals also were significantly better protected from live virus challenge. However, owing to limited number of animals in the study, it was not possible to assess the contribution of individual components.

Antigen processing and presentation is another important aspect of immunogenicity. Since DNA vaccines result in intracellular antigen expression de novo, the antigens have a propensity for MHC class I mediated presentation. For viruses such as dengue, where the antibody response is believed to be pivotal in protection, it may be beneficial to steer the antigen towards a class II MHC mediated presentation. Several reports have shown that the lysosome associated membrane protein (LAMP) and MHC II are co-localized in specialized cellular vesicles, called M-II-C, of the endosomal/lysosomal vesicular pathway of APC such as dendritic cells and macrophages[21-24]. The cytoplasmic domain of LAMP contains a putative lysosome/endosome translocation signal, and it has been shown that proteins, when encoded by plasmids engineered to contain a ER translocation signal sequence and the LAMP cytoplasmic domain, are directed to the MHC II compartment[25]. Lysosome trafficking of DNA vaccine encoded antigens fused to LAMP sequence, including human papilloma virus E7, HIV gp160, and carcino-embryonic antigen, was shown to result in a marked increase in both the cellular and the humoral immune responses in vaccinated mice[26-30]. In an effort to achieve this goal, we created a chimeric DNA vaccine construct 
in which the prM and a truncated E gene of dengue-2 virus were fused to the cytoplasmic tail of LAMP. Dengue antigen expressed from such a chimeric construct was shown to be co-localized in lysosomes with endogenous LAMP and class II MHC, and elicited higher antibody responses in vaccinated mice[31,32]. Addition of LAMP sequences resulted in significantly higher dengue-2 neutralizing antibodies. A similar increase was also seen when DNA without the LAMP sequence was administered with another plasmid containing murine GM-CSF gene. Mice immunized with LAMP chimera and the GM-CSF plasmid elicited a further 5-fold increase in neutralizing antibody response showing synergistic effects of lysosome targeting and GM-CSF. However, attempts to replicate this enhanced immune response with DNA constructs for other dengue serotypes were unsuccessful (unpublished data). Because of the increased immune response seen with the DEN-2 LAMP chimeric vaccine, we used this construct as the DEN-2 component of the tetravalent dengue vaccine.

Use of adjuvants to enhance immunogenicity of DNA vaccines has also received much attention. Many people have used immune function modulators such as GM-CSF, IL-2, CTLA-4, Fas-ligand, CD40 etc. as "adjuvants" to increase DNA vaccine induced immune responses [33-36]. Although such bioactive molecules show immune enhancing effects in mouse models, studies in large animal models are lacking. It is unlikely that bioactive molecules will become part of vaccine regimens intended for healthy individuals.

There is, however, pre-clinical evidence showing enhanced humoral responses of DNA vaccines in lipid-based formulations [37-39]. $\operatorname{Vaxfectin}^{\circledR}$, a cationic/neutral lipid 
combination developed by Vical [40] was used in a non-human primate study of a tetravalent dengue DNA vaccine. Vaxfectin ${ }^{\circledR}$-formulated vaccine led to a significant increase in both antibody titers and antibody longevity [41]. When challenged with DEN-2 live virus, animals vaccinated with formulated DNA had a mean duration of 0.75 days of viremia compared to 2 days for animals vaccinated with unformulated vaccine, and 3.3 days for control unvaccinated animals. In a subsequent pre-clinical safety study in New Zealand white rabbits, Vaxfectin ${ }^{\circledR}$ formulated dengue tetravalent DNA vaccine was well tolerated and elicited high titer virus neutralizing antibodies [42].

\section{Dengue DNA Vaccine Clinical Trials}

The DEN-1 monovalent DNA vaccine was immunogenic in non-human primate models and shown to provide significant protection against challenge with live DEN-1 virus. To show proof-of-principal in humans, we conducted a Phase 1 first-in-humans safety and immunogenicity clinical trial [43]. The trial was a dose escalation study involving 22 dengue naïve volunteers; 10 received the $1 \mathrm{mg}$ low dose DEN-1 DNA vaccine and 12 received the $5 \mathrm{mg}$ high dose. The vaccine was administered IM using the needle-free Biojector 2000 device. The monovalent DEN-1 DNA vaccine was safe with no serious adverse events reported. The most common symptom reported was mild tenderness at the vaccine administration site. The DNA vaccine elicited poor anti-dengue neutralizing antibody responses, with only five of 12 subjects in the high dose group responding. No subject immunized with $1 \mathrm{mg}$ developed neutralizing antibodies. T cell IFN $\gamma$ responses were much more pronounced, with $50 \%$ of subjects responding in the low dose group and 83\% responding in the high dose group. This Phase 1 trial demonstrated the monovalent 
DEN-1 DNA vaccine to be safe, well tolerated and geared toward eliciting $\mathrm{T}$ cell responses at the highest dose tested.

A tetravalent DNA vaccine consisting of plasmids expressing the prM $100 \% \mathrm{E}$ genes of each of the four serotypes demonstrated an enhanced anti-dengue neutralizing antibody response in nonhuman primates when formulated with a proprietary adjuvant Vaxfectin ${ }^{\circledR}$. The next step was to evaluate the formulated tetravalent vaccine in humans. An IND application was filed with the U. S. Food and Drug Administration to conduct a Phase 1 dose escalation study to evaluate safety, tolerability and immunogenicity (Clinical Trials.gov \#NCT01502358). A total of 40 subjects were enrolled, with 10 receiving the tetravalent dengue DNA vaccine (TVDV) alone at $1 \mathrm{mg}, 10$ receiving the TVDV at $1 \mathrm{mg}$ formulated in Vaxfectin ${ }^{\circledR}$ and 20 receiving TVDV at 2 mg formulated in Vaxfectin ${ }^{\circledR}$. The results of this trial are currently pending publication.

\section{Viral Vectored DNA Vaccines}

Delivery of DNA vaccines to target cells can be greatly improved by using viral vectors as vehicles. Viral vectors such as adenovirus and vaccinia virus, and alphavirus replicons such as VEE (Venezuelan equine encephalitis virus) replicons can deliver nucleic acid to cells by infection, which is much more efficient compared to transfection of cells in vivo. However, preexisting anti-vector antibodies in human populations may pose significant challenges. VEE replicon based vaccines are attractive because of their efficient infection of cells of dendritic morphology that migrate to lymph nodes[44] and the lack of widespread preexisting immunity to VEE virus in human populations. VEE virus replicon particles 
(VRP) have been used successfully to produce immune responses to a number of viral antigens in preclinical animal studies[45-47]. However, in a non-human primate study that compared a DEN-1 DNA vaccine with a VEE replicon vaccine expressing the same dengue antigens (prM 100\%E), no significant difference either in antibody response or in protection from viremia ( 0.66 and 0.75 mean days viremia, respectively, compared to 6.33 days for control unvaccinated group) upon challenge was noted [48]. In the same study, animals that were vaccinated by a heterologous prime-boost regimen (DNA prime followed by VEE replicon boost) showed about a two-fold increase in antibody titers and were completely protected from viremia upon challenge. A recent report by White et al [49] suggests that with this platform, VRP expressing $85 \% \mathrm{E}$ elicited better antibody responses compared to VRP containing prM 100\%E in rhesus macaques. Breakthrough viremia was reported when tetravalent VRP vaccine-immunized animals were challenged with DEN-1 or DEN-2 virus.

Adenovirus vectors have been widely used as DNA vaccine vehicles. Second generation complex adenovirus vectors with two promoter cassettes for expression of multiple antigens $[50,51]$ are especially attractive for dengue vaccine because of the need for a tetravalent dengue vaccine. After initially testing two bivalent dengue vaccine constructs for immunogenicity in mice $[52,53]$, a tetravalent dengue vaccine product based on two complex adenovirus vectors was tested in rhesus macaques. In this comprehensive study, all vaccinated animals $(n=24)$ elicited high titer neutralizing antibodies to all four dengue serotypes. Furthermore, when smaller groups were challenged with live dengue viruses at one month or six months post vaccination, complete protection against dengue- 1 and -3 viremia and partial protection against dengue-2 and -4 viremia was observed [54]. 
Because of issues raised regarding pre-existing immunity to adenovirus 5 and abrogation of vaccine-induced immune responses, as well as reports on the potential for increasing the risk of HIV infection among adenovirus positive subjects receiving an experimental adenovirus-vectored HIV vaccine, development of adenovirus 5 vectored dengue vaccines was discontinued.

\section{Conclusions}

Nucleic acid immunization is a vaccine platform that has demonstrated the ability to induce robust cellular and humoral immune responses in small animals and non-human primates. In fact, several DNA vaccines and therapies worldwide have been licensed for use in fish, animals and livestock [55]. To date, human clinical trials of this vaccine approach have been somewhat disappointing overall. Innovations in DNA vaccine delivery such as electroporation have shown great promise in eliciting the desired immune responses due to more efficient uptake and expression of DNA vaccine plasmids. This together with the advantages of stability and ease of production and genetic manipulation continue to make nucleic acid immunization an attractive vaccine platform.

\section{Acknowledgements}

The views expressed in this article are those of the authors and do not necessarily reflect the official policy or position of the Department of the Navy, Department of Defense or the U.S. Government. The authors are U. S. Government employees and this work was prepared as part of their official duties. Title 17 U.S.C 105 provides that "Copyright 
protection under this title is not available for any work of the U.S. government.' Title 17 U.S.C 101 defines a Government work as a work prepared by U. S. Government members as part of that person's official duties.

Potential conflicts of interest. Kevin Porter and Kanakatte Raviprakash are co-patent holders on US Patent No. US 6,455,509 B1, Title "Dengue Nucleic Acid Vaccines that Induce Neutralizing Antibodies".

\section{References}

[1] Simmons CP, Farrar JJ, Nguyen v V, Wills B. Dengue. The New England journal of medicine. 2012;366:1423-32.

[2] Kochel T, Wu SJ, Raviprakash K, Hobart P, Hoffman S, Porter K, et al. Inoculation of plasmids expressing the dengue-2 envelope gene elicit neutralizing antibodies in mice. Vaccine. 1997;15:547-52.

[3] Raviprakash K, Kochel T], Ewing D, Simmons M, Phillips I, Hayes CG, et al. Immunogenicity of dengue virus type 1 DNA vaccines expressing truncated and full length envelope protein. Vaccine. 2000;18:2426-34.

[4] Konishi E, Kosugi S, Imoto J. Dengue tetravalent DNA vaccine inducing neutralizing antibody and anamnestic responses to four serotypes in mice. Vaccine. 2006;24:2200-7. [5] Konishi E, Yamaoka M, Kurane I, Mason PW. A DNA vaccine expressing dengue type 2 virus premembrane and envelope genes induces neutralizing antibody and memory B cells in mice. Vaccine. 2000;18:1133-9. 
[6] Costa SM, Paes MV, Barreto DF, Pinhao AT, Barth OM, Queiroz JL, et al. Protection against dengue type 2 virus induced in mice immunized with a DNA plasmid encoding the non-structural 1 (NS1) gene fused to the tissue plasminogen activator signal sequence. Vaccine. 2006;24:195-205.

[7] Lu H, Xu XF, Gao N, Fan DY, Wang J, An J. Preliminary evaluation of DNA vaccine candidates encoding dengue-2 prM/E and NS1: their immunity and protective efficacy in mice. Mol Immunol. 2013;54:109-14.

[8] Zheng Q, Fan D, Gao N, Chen H, Wang J, Ming Y, et al. Evaluation of a DNA vaccine candidate expressing prM-E-NS1 antigens of dengue virus serotype 1 with or without granulocyte-macrophage colony-stimulating factor (GM-CSF) in immunogenicity and protection. Vaccine. 2011;29:763-71.

[9] Kochel TJ, Raviprakash K, Hayes CG, Watts DM, Russell KL, Gozalo AS, et al. A dengue virus serotype-1 DNA vaccine induces virus neutralizing antibodies and provides protection from viral challenge in Aotus monkeys. Vaccine. 2000;18:3166-73. [10] Raviprakash K, Porter KR, Kochel TJ, Ewing D, Simmons M, Phillips I, et al. Dengue virus type 1 DNA vaccine induces protective immune responses in rhesus macaques. J Gen Virol. 2000;81 Pt 7:1659-67.

[11] Blair PJ, Kochel TJ, Raviprakash K, Guevara C, Salazar M, Wu SJ, et al. Evaluation of immunity and protective efficacy of a dengue-3 pre-membrane and envelope DNA vaccine in Aotus nancymae monkeys. Vaccine. 2006;24:1427-32.

[12] Putnak R, Fuller J, VanderZanden L, Innis BL, Vaughn DW. Vaccination of rhesus macaques against dengue-2 virus with a plasmid DNA vaccine encoding the viral premembrane and envelope genes. Am J Trop Med Hyg. 2003;68:469-76. 
[13] Apt D, Raviprakash K, Brinkman A, Semyonov A, Yang S, Skinner C, et al. Tetravalent neutralizing antibody response against four dengue serotypes by a single chimeric dengue envelope antigen. Vaccine. 2006;24:335-44.

[14] Raviprakash K, Apt D, Brinkman A, Skinner C, Yang S, Dawes G, et al. A chimeric tetravalent dengue DNA vaccine elicits neutralizing antibody to all four virus serotypes in rhesus macaques. Virology. 2006;353:166-73.

[15] Corr M, von Damm A, Lee DJ, Tighe H. In vivo priming by DNA injection occurs predominantly by antigen transfer. J Immunol. 1999;163:4721-7.

[16] Raviprakash KaP, KR. Needle-Free Injection of DNA Vaccines. In: Saltzman WM SHaBJ, editor. DNA Vaccines: Methods and Protocols. 2 ed. New Jersey, USA: Humana; 2006. p. 839.

[17] Manam S, Ledwith BJ, Barnum AB, Troilo PJ, Pauley CJ, Harper LB, et al. Plasmid DNA vaccines: tissue distribution and effects of DNA sequence, adjuvants and delivery method on integration into host DNA. Intervirology. 2000;43:273-81.

[18] Kojima Y, Xin KQ, Ooki T, Hamajima K, Oikawa T, Shinoda K, et al. Adjuvant effect of multi-CpG motifs on an HIV-1 DNA vaccine. Vaccine. 2002;20:2857-65.

[19] Qiu JT, Chang TC, Lin CT, Chen YM, Li FQ, Soong YK, et al. Novel codon-optimized GMCSF gene as an adjuvant to enhance the immunity of a DNA vaccine against HIV-1 Gag. Vaccine. 2007;25:253-63.

[20] Raviprakash K, Ewing D, Simmons M, Porter KR, Jones TR, Hayes CG, et al. Needle-free Biojector injection of a dengue virus type 1 DNA vaccine with human immunostimulatory sequences and the GM-CSF gene increases immunogenicity and protection from virus challenge in Aotus monkeys. Virology. 2003;315:345-52. 
[21] Harding CV, Geuze HJ. Immunogenic peptides bind to class II MHC molecules in an early lysosomal compartment. J Immunol. 1993;151:3988-98.

[22] Kleijmeer MJ, Ossevoort MA, van Veen CJ, van Hellemond JJ, Neefjes JJ, Kast WM, et al. MHC class II compartments and the kinetics of antigen presentation in activated mouse spleen dendritic cells. J Immunol. 1995;154:5715-24.

[23] Peters PJ, Raposo G, Neefjes JJ, Oorschot V, Leijendekker RL, Geuze HJ, et al. Major histocompatibility complex class II compartments in human B lymphoblastoid cells are distinct from early endosomes. J Exp Med. 1995;182:325-34.

[24] Turley SJ, Inaba K, Garrett WS, Ebersold M, Unternaehrer J, Steinman RM, et al. Transport of peptide-MHC class II complexes in developing dendritic cells. Science. 2000;288:522-7.

[25] Guarnieri FG, Arterburn LM, Penno MB, Cha Y, August JT. The motif Tyr-X-Xhydrophobic residue mediates lysosomal membrane targeting of lysosome-associated membrane protein 1. J Biol Chem. 1993;268:1941-6.

[26] Lin KY, Guarnieri FG, Staveley-O'Carroll KF, Levitsky HI, August JT, Pardoll DM, et al. Treatment of established tumors with a novel vaccine that enhances major histocompatibility class II presentation of tumor antigen. Cancer Res. 1996;56:21-6. [27] Nair SK, Boczkowski D, Morse M, Cumming RI, Lyerly HK, Gilboa E. Induction of primary carcinoembryonic antigen (CEA)-specific cytotoxic T lymphocytes in vitro using human dendritic cells transfected with RNA. Nat Biotechnol. 1998;16:364-9. [28] Rowell JF, Ruff AL, Guarnieri FG, Staveley-O'Carroll K, Lin X, Tang J, et al. Lysosomeassociated membrane protein-1-mediated targeting of the HIV-1 envelope protein to an 
endosomal/lysosomal compartment enhances its presentation to MHC class II-restricted T cells. J Immunol. 1995;155:1818-28.

[29] Ruff AL, Guarnieri FG, Staveley-O'Carroll K, Siliciano RF, August JT. The enhanced immune response to the HIV gp160/LAMP chimeric gene product targeted to the lysosome membrane protein trafficking pathway. J Biol Chem. 1997;272:8671-8.

[30] Wu TC, Guarnieri FG, Staveley-O'Carroll KF, Viscidi RP, Levitsky HI, Hedrick L, et al. Engineering an intracellular pathway for major histocompatibility complex class II presentation of antigens. Proc Natl Acad Sci U S A. 1995;92:11671-5.

[31] Lu Y, Raviprakash K, Leao IC, Chikhlikar PR, Ewing D, Anwar A, et al. Dengue 2 PreME/LAMP chimera targeted to the MHC class II compartment elicits long-lasting neutralizing antibodies. Vaccine. 2003;21:2178-89.

[32] Raviprakash K, Marques E, Ewing D, Lu Y, Phillips I, Porter KR, et al. Synergistic neutralizing antibody response to a dengue virus type 2 DNA vaccine by incorporation of lysosome-associated membrane protein sequences and use of plasmid expressing GM-CSF. Virology. 2001;290:74-82.

[33] Chen Q, Zhu G, Wang R, Zhang J, He G. Adjuvant effect of CD40 on H5N1 DNA vaccine in mice. Arch Virol. 2014;159:1359-64.

[34] Yang H, Cao S, Huang X, Liu J, Tang Y, Wen X. Intragastric administration of attenuated Salmonella typhimurium harbouring transmissible gastroenteritis virus (TGEV) DNA vaccine induced specific antibody production. Vaccine. 2009;27:5035-40.

[35] Nimal S, Thomas MS, Heath AW. Fusion of antigen to Fas-ligand in a DNA vaccine enhances immunogenicity. Vaccine. 2007;25:2306-15. 
[36] Yin Y, Wu C, Song J, Wang J, Zhang E, Liu H, et al. DNA immunization with fusion of CTLA-4 to hepatitis B virus (HBV) core protein enhanced Th2 type responses and cleared HBV with an accelerated kinetic. PLoS One. 2011;6:e22524.

[37] Hartikka J, Bozoukova V, Ferrari M, Sukhu L, Enas J, Sawdey M, et al. Vaxfectin enhances the humoral immune response to plasmid DNA-encoded antigens. Vaccine. 2001;19:1911-23.

[38] Margalith M, Vilalta A. Sustained protective rabies neutralizing antibody titers after administration of cationic lipid-formulated pDNA vaccine. Genet Vaccines Ther. 2006;4:2. [39] Reyes L, Hartikka J, Bozoukova V, Sukhu L, Nishioka W, Singh G, et al. Vaxfectin enhances antigen specific antibody titers and maintains Th1 type immune responses to plasmid DNA immunization. Vaccine. 2001;19:3778-86.

[40] Sullivan SM, Doukas J, Hartikka J, Smith L, Rolland A. Vaxfectin: a versatile adjuvant for plasmid DNA- and protein-based vaccines. Expert Opin Drug Deliv. 2010;7:1433-46. [41] Porter KR, Ewing D, Chen L, Wu SJ, Hayes CG, Ferrari M, et al. Immunogenicity and protective efficacy of a vaxfectin-adjuvanted tetravalent dengue DNA vaccine. Vaccine. 2012;30:336-41.

[42] Raviprakash K, Luke T, Doukas J, Danko J, Porter K, Burgess T, et al. A dengue DNA vaccine formulated with Vaxfectin(R) is well tolerated, and elicits strong neutralizing antibody responses to all four dengue serotypes in New Zealand white rabbits. Hum Vaccin Immunother. 2012;8:1764-8.

[43] Beckett CG, Tjaden J, Burgess T, Danko JR, Tamminga C, Simmons M, et al. Evaluation of a prototype dengue-1 DNA vaccine in a Phase 1 clinical trial. Vaccine. 2011;29:960-8. 
[44] MacDonald GH, Johnston RE. Role of dendritic cell targeting in Venezuelan equine encephalitis virus pathogenesis. J Virol. 2000;74:914-22.

[45] Lee JS, Pushko P, Parker MD, Dertzbaugh MT, Smith LA, Smith JF. Candidate vaccine against botulinum neurotoxin serotype A derived from a Venezuelan equine encephalitis virus vector system. Infect Immun. 2001;69:5709-15.

[46] Schultz-Cherry S, Dybing JK, Davis NL, Williamson C, Suarez DL, Johnston R, et al. Influenza virus (A/HK/156/97) hemagglutinin expressed by an alphavirus replicon system protects chickens against lethal infection with Hong Kong-origin H5N1 viruses. Virology. 2000;278:55-9.

[47] Wilson JA, Hart MK. Protection from Ebola virus mediated by cytotoxic T lymphocytes specific for the viral nucleoprotein. J Virol. 2001;75:2660-4.

[48] Chen L, Ewing D, Subramanian H, Block K, Rayner J, Alterson KD, et al. A heterologous DNA prime-Venezuelan equine encephalitis virus replicon particle boost dengue vaccine regimen affords complete protection from virus challenge in cynomolgus macaques. J Virol. 2007;81:11634-9.

[49] White LJ, Sariol CA, Mattocks MD, Wahala MPBW, Yingsiwaphat V, Collier ML, et al. An alphavirus vector-based tetravalent dengue vaccine induces a rapid and protective immune response in macaques that differs qualitatively from immunity induced by live virus infection. J Virol. 2013;87:3409-24.

[50] Wang D, Hevey M, Juompan LY, Trubey CM, Raja NU, Deitz SB, et al. Complex adenovirus-vectored vaccine protects guinea pigs from three strains of Marburg virus challenges. Virology. 2006;353:324-32. 
[51] Wang D, Raja NU, Trubey CM, Juompan LY, Luo M, Woraratanadharm J, et al. Development of a cAdVax-based bivalent ebola virus vaccine that induces immune responses against both the Sudan and Zaire species of Ebola virus. J Virol. 2006;80:273846.

[52] Holman DH, Wang D, Raviprakash K, Raja NU, Luo M, Zhang J, et al. Two complex, adenovirus-based vaccines that together induce immune responses to all four dengue virus serotypes. Clin Vaccine Immunol. 2007;14:182-9.

[53] Raja NU, Holman DH, Wang D, Raviprakash K, Juompan LY, Deitz SB, et al. Induction of bivalent immune responses by expression of dengue virus type 1 and type 2 antigens from a single complex adenoviral vector. Am J Trop Med Hyg. 2007;76:743-51.

[54] Raviprakash K, Wang D, Ewing D, Holman DH, Block K, Woraratanadharm J, et al. A tetravalent dengue vaccine based on a complex adenovirus vector provides significant protection in rhesus monkeys against all four serotypes of dengue virus. J Virol. 2008;82:6927-34.

[55] Kutzler MA, Weiner DB. DNA vaccines: ready for prime time? Nat Rev Genet. 2008;9:776-88. 
Table 1 U. S. Navy DNA and Virus Vectored Vaccine Approaches

\begin{tabular}{|c|c|c|c|c|}
\hline Method & Pre-Clinical & Clinical & Issues & References \\
\hline $\begin{array}{l}\text { Monovalent DNA. } \\
\text { IM/ID needle injection. }\end{array}$ & Immunogenic in mice and NHP & - & - & {$[1-4][5]$} \\
\hline $\begin{array}{l}\text { Monovalent DNA. } \\
\text { Needle-free Biojector } \\
\text { injection, IM. }\end{array}$ & $\begin{array}{l}\text { Increased immunogenicity in } \\
\text { NHP. }\end{array}$ & $\begin{array}{l}\text { Weakly immunogenic. } \\
\text { Weak antibody response } \\
\text { but better T cell responses } \\
\text { in a subset of individuals. }\end{array}$ & $\begin{array}{l}\text { Antibody responses present only in } \\
\text { high dose group ( } 5 \mathrm{mg} \text { DNA). }\end{array}$ & {$[6,7]$} \\
\hline $\begin{array}{l}\text { Tetravalent DNA; single } \\
\text { plasmid shuffled DNA } \\
\text { approach. }\end{array}$ & $\begin{array}{l}\text { Tetravalent immune responses } \\
\text { in mice; weaker tetravalent } \\
\text { responses in NHP. }\end{array}$ & - & - & {$[8,9]$} \\
\hline $\begin{array}{l}\text { LAMP chimera DNA } \\
\text { approach }\end{array}$ & $\begin{array}{l}\text { Increased immunogenicity for } \\
\text { dengue-2 DNA vaccine in mice. }\end{array}$ & $\begin{array}{l}\text { Part of the tetravalent DNA } \\
\text { vaccine cocktail. }\end{array}$ & $\begin{array}{l}\text { Effect on dengue- } 2 \text { could not be } \\
\text { replicated for other serotypes. }\end{array}$ & {$[10,11]$} \\
\hline $\begin{array}{l}\text { Tetravalent DNA (mix of } 4 \\
\text { monovalent DNAs) + } \\
\text { Vaxfectin, IM, Biojector }\end{array}$ & $\begin{array}{l}\text { Increased antibody response } \\
\text { to all } 4 \text { serotypes in NHP. }\end{array}$ & - & - & {$[12]$} \\
\hline $\begin{array}{l}\text { Tetravalent DNA (mix of } 4 \\
\text { monovalent DNAs) + } \\
\text { Vaxfectin, IM, Needle }\end{array}$ & $\begin{array}{l}\text { Immunogenicity and safety } \\
\text { demonstrated in white rabbits. }\end{array}$ & $\begin{array}{l}\text { Clinical trial completed. } \\
\text { Final report and } \\
\text { manuscript in progress. }\end{array}$ & $\begin{array}{l}\text { Maximum DNA that could be } \\
\text { formulated and administered was } 2 \\
\mathrm{mg} / \text { dose. }\end{array}$ & {$[13]$} \\
\hline $\begin{array}{l}\text { VEE replicon particle (VRP) } \\
\text { vaccines. }\end{array}$ & $\begin{array}{l}\text { Monovalent dengue-1 \& } \\
\text { tetravalent mixtures } \\
\text { immunogenic in mice and } \\
\text { NHP. Better when used for } \\
\text { boosting }\end{array}$ & - & $\begin{array}{l}\text { Difficult to produce large quantities. } \\
\text { Generating replication competent } \\
\text { virus is a concern. }\end{array}$ & {$[14]$} \\
\hline $\begin{array}{l}\text { Adenovirus vectored } \\
\text { vaccines. }\end{array}$ & $\begin{array}{l}\text { Tetravalent formulation } \\
\text { immunogenic in mice and } \\
\text { protective in NHP challenge } \\
\text { studies. }\end{array}$ & - & $\begin{array}{l}\text { Safety concerns about Ad-5 based } \\
\text { vectors. }\end{array}$ & {$[15-17]$} \\
\hline
\end{tabular}

NHP=nonhuman primates; LAMP=lysosome associated membrane protein; Ad-5=adenovirus serotype 5 